\title{
IR thermography analysis for ceramic themo-physical property evaluation
}

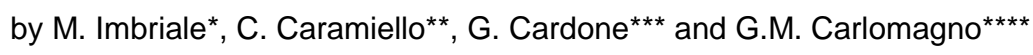 \\ *Dept. of Aerospace Engineering, University of Naples, via Claudio 21 - 80125 Napoli, Italy, \\ michele.imbriale@unina.it \\ ** E.M.A. S.p.A, Zona Industriale ASI 83040 Morra De Sanctis (AV), caramiello@emaht.com \\ $\star \star \star$ Dept. of Aerospace Engineering, University of Naples, via Claudio $21-80125$ Napoli, Italy, \\ gcardone@unina.it \\ ${ }^{\star \star \star \star D}$ Dept. of Aerospace Engineering, University of Naples, p.le tecchio 80- 80125 Napoli, Italy, \\ carmagno@unina.it
}

\begin{abstract}
Investment casting is one of the oldest manufacturing processes, dating back thousands of years, in which molten metal is poured into an expendable ceramic mold. It's one-to-one process: one ceramic shell patterns produces one part. In investment casting ceramic shells are formed by using a wax pattern (a disposable piece in the shape of the desired part). The pattern is surrounded, or "invested", into ceramic slurry that hardens into a furnace. Investment casting is often referred to as "lost-wax casting" because the wax pattern is melted out of the shell after it has been formed. Loxwax processes are one-to-one (one pattern creates one part), which increases production time and costs relative to other casting processes. However, since the mold is destroyed during the process, parts with complex geometries and intricate details can be created.

Shell heating and cooling involve the not well-defined shell's thermo-physical properties; the ceramic shell thermo-physical properties deserve particular attention on because the shell represent the interface between super-alloy and the ambient during pattern cooling. Consequently knowing shell properties helps to simulate the heat transfer evolution to improve process yield. In heat transfer processes are involved a high number of parameters, in particular the works deals with convective heat transfer coefficient, ceramic conductivity and volumetric specific heat assessment.

Of course, since the EMA investment casting involves a wide range of temperature, will be take in account the property temperature dependence Moreover, since investment casting is one-to-one process, the shell is always different, hence the new measurement method has to be quickly fast and robust also to use it as a statistical check.

Since last century many thermo-physical properties measurement techniques were developed. In particular, for thermal diffusivity the flash and laser flash method [1-4] are widely studied and used, but many other methods are possible: photo-thermal [5], lock-in thermography [6-9], laser interferometer [10] or other methods based on a continuous heat source [11]. Also some comparisons among different methods are reported in literature [12]. All these methods are contactless and it's often used an infrared detector to evaluate surface temperature, but they are studied and tested at relatively low temperature and in little temperature ranges. Moreover nobody had ever developed a measurement technique by means of which it is simultaneously possible to assess convective heat transfer coefficient, thermal conductivity and volumetric specific heat (consequently thermal diffusivity) and to evaluate the temperature dependence of this properties.

An unsteady technique based on IR thermography measurements is proposed to quickly evaluate ceramic shell thermo-physical properties. The method has been developed for low-diffusivity materials as well as for the ceramic materials self-made by E.M.A. S.p.A. for gas turbine blade manufacturing by investment casting. A ceramic sample is heated up to about $1000^{\circ} \mathrm{C}$ and cooled in quiet air at room temperature while an infrared camera and a pyrometer detect the time dependence surface temperature.
\end{abstract}

Cooling of an infinite isotropic slab can be described by boundary balance for unit area of heat transfer:

$$
\rho c \frac{s}{2} \frac{d \bar{T}}{d t}=-h \cdot\left(T_{s}-T_{\infty}\right)-\sigma \varepsilon\left(T_{s}^{4}-T_{\infty}^{4}\right)
$$

where $\sigma=5.67 \cdot 10^{-8} \frac{W}{m^{2} K^{4}}$ is Stefan-Boltzmann constant, $\rho, c, \varepsilon$ are respectively the slab material density, specific heat and emissivity, $s$ is slab thickness, $h$ convective heat transfer coefficient, $\bar{T}, T_{s}$ and $T_{\infty}$ are respectively mean temperature along thickness, heat transfer surface temperature and room temperature. In fig.1 is shown the ceramic slab sketch in cooling.

Making some mathematical steps and reasonable approximations [13] the equation (1) become:

$$
\frac{d\left[T_{S}\left(t^{*}\right)\right]}{d t}=-\chi \cdot\left(T_{s}(t)-T_{\infty}\right)-\xi \cdot\left(T_{S}(t)^{4}-T_{\infty}^{4}\right)
$$

where $\chi=\frac{2 h}{\rho c s}, \xi=\frac{2 \sigma \varepsilon}{\rho c s}, \bar{T}(t) \cong T_{s}\left(\mathrm{t}^{*}\right)$ and $t^{*}=t-\frac{s^{2}}{3 a}$. 
Comparing experimental data against the theoretical curve and minimizing the quadratic difference between them it's possible evaluate the unknown parameters $\chi, \xi$, and so thermal diffusivity $a$ ( $\rho c$ product) conductivity $k$ and convective heat transfer coefficient $h$.

The experiments are carried out in E.M.A. S.p.A. because both its furnaces are able to reach nearly $1000{ }^{\circ} \mathrm{C}$ and material specimen is unique and made only by EMA.

Test specimens were manufactured to simulate the cooling process of a infinite slab (two dimension much greater than other one). So we can neglect conduction loss in two directions and make use of equation (2).

Ceramic slab is heated up to about $1000^{\circ} \mathrm{C}$ in EMA furnace and cooled in quiet air at room temperature. Experimental cooling curves are tested in ranges greater than fifty degrees in which we consider constant all thermophysical properties and are compared with theoretical curves seen above. In this way we can obtain the parameters at high temperature and temperature dependence in a wide temperature range.

The present work shows how it's possible to make easier the monitoring of the main ceramic shell thermophysical property necessary for investment casting, making use of instruments normally present investment casting industries.

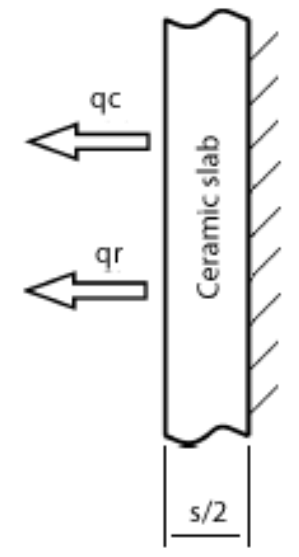

Fig. 1. Ceramic Slab

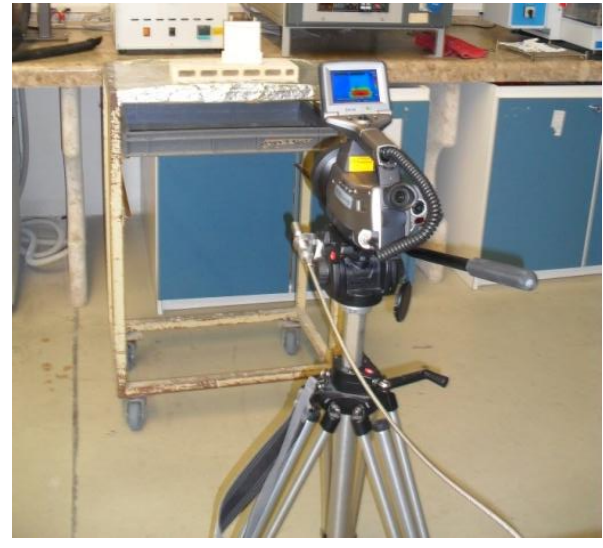

Fig. 2. Experimental Apparatus

\section{REFERENCES}

[1] A.J.Angstrom, Philos. Mag. 25, 130, 1863.

[2] H.S. Carslaw, J.C.Jaeger, Conduction of heat in solids, Clarendon, Oxford, 1959.

[3] W.J.Parker, R.J.Jenkins, C.P.Butler, G.L.Abbott, J.Appl.Phys.32, 1679-1684, 1961.

[4] F.Cernuschi, A.Russo, L.Lorenzoni, A.Figari, In-plane thermal diffusivity evaluation by infrared thermography, Review of scientific instruments, Vol. 72, n.10, 2001.

[5] M.Bertolotti, R.Li Voti, G.Liakhou, C.Sibilia, On the photodeflection method applied to low thermal diffusivity measurements, Rev. Sci. Instr. 64(6), 1576-1583, 1993.

[6] C.Meola, G.M.Carlomagno, A.Squillace, G.Giorleo, Non-Destructive control of industrial materials by means of lock-in thermography, Measurement Science and Technology, 13 1583-1590, 2002.

[7] S.Marinetti, P.G.Bison, E.Grinzato, A.Muscio, Thermal diffusivity measurement of stainless steel by periodic heating technique, AITA, 316-321, 1999.

[8] A.Muscio, E.Grinzato, The lock-in heating-cooling method for the measurement of the thermal diffusivity of solid materials, Heat Transfer Engineering,23 (2), 44-52, 2002.

[9] A.Muscio, P.G. Bison, S.Marinetti, E.Grinzato, Thermal diffusivity measurement in slabs using harmonic and one-dimensional propagation of thermal waves, Intern. Journ. of Thermal Science 43, 453-463, 2004.

[10] N.Sparvieri, E.Penco, C.Sibilia, M.Bertolotti, G.Suber, A.Ferrari, Mater.Lett.5, 449-452, 1987.

[11] J.M. Laskar, S.Bagavathiappan, M.Sardar, T.Jayakumar, J.Philip, B.Raj, Measurement of thermal diffusivity of solids using infrared thermography, Mater. Lett. 62, 2740-2742, 2008.

[12] P.G. Bison, S.Marinetti, A.Mazzoldi, E.Grinzato, C.Bressan, Cross-comparison of thermal diffusivity measurements by thermal methods, Infrared Physics \& Thechnology 43, 127-132, 2002.

[13] D.J.Baines, A comparative theoretical evaluation of five commonly used types of unsteady heat flux sensors, Report HAS 27, 1970. 